\title{
NOTAS SOBRE EL VÍNCULO ENTRE INSTITUCIONES, DESIGUALDADES SOCIALES Y SALUD
}

\section{NOTES ON THE LINK BETWEEN INSTITUTIONS, SOCIAL INEQUALITIES AND HEALTH}

\author{
Mauricio López Ruiz*
}

RESUMEN

Debates como el de los determinantes sociales de la salud, desarrollados en el marco de lo que Nancy Krieger denomina epidemiología social, han permitido legitimar el estudio de las instituciones sociales como un factor relevante para comprender la configuración dispar de patrones de mortalidad y morbilidad en distintos grupos poblacionales. El presente artículo resume argumentos centrales que se desarrollan en el campo de la salud pública sobre este tema, con el fin de contrastar sus aportes analíticos en relación con las formas tradicionales de entender la salud y la enfermedad, basadas en encuadres biomédicos e individualizados.

PALABRAS CLAVE: SALUD PÚBLICA * DESIGUALDAD * EPIDEMIOLOGÍA SOCIAL

\section{ABSTRACT}

The debate about social determinants of health categorized by Nancy Krieger as part of the so called social epidemiology have helped to legitimize the study of social institutions as an important factor to understand the creation of uneven morbidity and mortality patterns among populations. This article offers a summary of key arguments on social determinants of health, in order to compare their contributions to the public health field when compared to biomedical and individualizing ideas about health and illness.

KEYWORDS: PUBLIC HEALTH * INEQUALITY * SOCIAL EPIDEMIOLOGY 


\section{INTRODUCCIÓN}

La salud pública, en tanto campo del saber, se ha configurado al calor de los intereses y conocimientos que día tras día son aportados por actores públicos y privados, tales como, compañías farmacéuticas, organismos internacionales, colegios profesionales, centros universitarios o agencias estatales. Siguiendo a Saracci (2010), la mayor parte de la producción intelectual generada por estos actores suele girar en torno a tres grandes tipos de investigaciones. Primero, estudios observacionales, surgidos ya sea de la labor de diagnóstico que se llevan acabo sobre mortalidad y morbilidad o bien, de la realización de encuestas o estudios trasversales y longitudinales sobre distintos temas de salud. En segundo lugar, intervenciones cuyo objetivo consiste en tratar y prevenir enfermedades, como por ejemplo, cuando equipos de profesionales en salud prueban la efectividad de vacunas en ciertas poblaciones. Por último, investigaciones de tipo experimental desarrolladas en laboratorios, las cuales le permiten a especialistas en ciencias biomédicas, la manipulación de organismos vivientes.

Los estudios observacionales, por mucho los más extendidos en el campo de la salud pública, han sido decisivos para el desarrollo contemporáneo de políticas en materia de prevención y promoción de la salud. Al estar usualmente basados en datos cuantificables producidos por medio de investigaciones o controles poblacionales relativos a la salud, dichos estudios informan acerca de patrones generalizados de morbilidad $y$ mortalidad que caracterizan a un conjunto social. Asimismo, la reconstrucción de estos patrones descansa en mayor o menor grado en la epidemiología, disciplina encargada de analizar "la presencia $y$ distribución de estados o eventos relativos a la salud en poblaciones concretas", así como de explicar el desarrollo de dichos estados o eventos (Porta, 2008: 81). Como es de suponerse, dependiendo del encuadre teórico que haya sido utilizado para enmarcar el quehacer investigativo de las y los epidemiólogos, así será la forma en que se dará sentido y evaluará el vínculo entre salud, enfermedades y poblaciones humanas.
En relación con este último tema, vale la pena recordar que a la fecha, el encuadre epidemiológico predominante se enfoca en el estudio de procesos biológicos y de la conducta individual, empatando de esta manera con el trabajo que se realiza en la medicina clínica. En ambos casos se utilizan distinciones "biomédicas" entre lo normal y lo patológico, en donde la enfermedad es causada por anomalías de tipo genético, químico o fisiológico "que afectan a funciones o estructuras del organismo humano" (Martínez, 2008: 50) ${ }^{1}$. Las anomalías citadas en el anterior párrafo, tienden a ser entendidas como algo potenciado por ciertos "factores de riesgo", noción ligada ya sea a "un aspecto de la conducta personal", a una "característica congénita" o bien, a algún "tipo de exposición ambiental" que se ha demostrado perjudicial para el bienestar de los seres humanos (Porta, 2008: 218). Cuando se habla de factores de riesgo en relación a aspectos de la conducta personal, usualmente se recurre a la vieja idea de "estilos de vida", para indicar aquella serie de decisiones (desatinadas en este caso) que las personas se empeñan en adoptar y convertir en hábitos, tales como, fumar tabaco, tomar alcohol en exceso, usar drogas ilícitas o no protegerse adecuadamente en sus prácticas sexuales (Krieger, 2012: 148).

Como puede notarse, esta idea de estilos de vida como factor de riesgo da lugar a relatos $y$ representaciones individualizadas acerca de la salud $y$ la enfermedad ${ }^{2}$. Lo anterior conlleva, entre otras cosas, a que la información de primera mano producida por las y los epidemiólogos siga tratándose como una mera sumatoria de datos clínicos personales agrupada en torno a variables como, edad, sexo o tipo de ocupación. Si bien es cierto, gracias al uso de técnicas estadísticas, esta información hace referencia a patrones generales de morbilidad $y$ mortalidad experimentados por diferentes grupos poblaciones, los datos resultantes no dejan de ser

$1 \quad$ Para una discusión sobre el surgimiento y significado del término biomedicina, véase Martínez, 2008, capítulo 2 y Krieger, 2012, capítulo 5.

2 Para un enfoque menos individualizado sobre el tema de estilos de vida, véase Cockerham, 2008 y 2012 . 
vistos como compilaciones de datos agregados. En este sentido, una variable como sexo no funciona en términos de un factor explicativo, como una variable independiente dentro de un modelo de análisis destinado a examinar las causas de dichos patrones de mortalidad y morbilidad, sino más bien, como una categoría que será de utilidad para ordenar datos sobre estados de salud. Al final de cuentas —esto quizá sea lo más relevante para efectos del presente artículo - la idea de estilos de vida como factor de riesgo presume la mayoría de las veces, a un individuo racional cuya condición de salud o enfermedad constituye la sumatoria de sus elecciones de vida.

A pesar del peso que continúan teniendo los encuadres biomédico e individualizados a la hora de analizar y evaluar estados de salud, en la actualidad, es posible distinguir perspectivas complementarias, las cuales postulan que dichos estados tienen su origen en dinámicas institucionales, cuya compresión no se reduce al examen tradicional de factores de riesgo $y$ estilos de vida aislados. Con el fin de profundizar este argumento, se revisarán las ideas establecidas en el campo de lo que Nancy Krieger (2012) ha denominado 'epidemiología social', centrando la atención sobre todo en trabajos que han sido sustentados en el estudio de la desigualdad social.

\section{DE FACTORES DE RIESGO A CAUSAS FUNDAMENTALES: LOS 'GRADIENTES' SOCIALES Y EL 'REDESCUBRIMIENTO' DE LO INSTITUCIONAL}

El encuadre biomédico que subyace en la noción de factores de riesgo (especialmente, cuando es traducida en términos de estilos de vida), ha ayudado no solo a explicar el surgimiento y desarrollo de estados de salud, sino también, ha contribuido a delimitar ámbitos morales de responsabilidad en el campo de la salud pública. Una vez descartada la intermediación de factores de riesgo ajenos a la voluntad individual, como por ejemplo, el heredar algún tipo de padecimiento o el no conocer que se estaba expuesto a un riesgo ambiental, la aparición de ciertas enfermedades tiende a ubicarse en la esfera de la responsabilidad personal. En otras palabras, lo que resulta más cómodo en estos casos es culpar a las personas por sus infortunios. Se dirá por ejemplo de aquella persona con problemas coronarios, que tuvo que haber meditado dos veces sobre sus hábitos alimenticios y haberle hecho caso a su médico sobre la conveniencia de salir aunque sea a trotar una media hora al día. Asimismo, se dirá del profesional en salud pública, que tuvo que haber diseñado campañas para enseñarle a la gente desde la infancia, sobre las bondades del comer saludablemente $y$ hacer ejercicio, campañas capaces de educarles y disciplinarles, para que hubiesen aprovechado los avances que ofrece la medicina moderna y pudieran al fin, ya de grandes, ejercer el cuido de sí mismos.

Desde luego, el establecimiento de este tipo de aseveraciones no es descabellado. Si hay algo que nos cuesta captar a las personas comunes y corrientes, como usted y como yo, es la imbricación que acontece a diario entre nuestras biografías personales y nuestras biografías colectivas. Por ello, al final siempre resulta más sencillo elaborar explicaciones basadas en lo inmediato, en lo que sale a flote en la superficie, en este caso, las elecciones adoptadas a conciencia por una persona. Sin embargo, ¿pueden los estados de salud que exhibe una población ser analizados como la mera sumatoria de decisiones personales?, ¿responden únicamente a factores de riesgo aislados y aleatorios, como por ejemplo, aquellos de origen ambiental o genético? Actualmente, se cuenta con evidencia empírica que apunta hacia una dirección alternativa, una que permite postular que distintos tipos de arreglos institucionales influyen en el desarrollo de distintos estados de salud, en especial, cuando los mismos producen o reproducen procesos de desigualdad social ${ }^{3}$.

Con el fin de dar cuenta de lo anterior, uno de los términos analíticos más apropiados que ha surgido en el campo de la salud públi-

$3 \quad$ El vínculo entre desigualdad social, enfermedad y salud, no es nuevo. De una u otra manera ha estado presente, entre otros, en el influyente trabajo llevado a cabo por Rudolf Virchow en el siglo XIX, así como en el quehacer que un siglo después desarrollarían salubristas anglosajones o la medicina social latinoamericana. 
ca para criticar a la noción de factor de riesgo (tal y como fueron definidos anteriormente) es el de "causas fundamentales", acuñado por Link y Phelan (1995 y 1996). Desde su punto de vista, las sociedades dan lugar tanto a patrones compartidos de salud y enfermedad, como a la manera en que los mismos serán explicados y experimentados por las personas. Los factores de riesgo en realidad constituirían "causas superficiales" que son producidos y reproducidos por medio de procesos institucionales mayores, los cuales requieren ser analizados y entendidos (Link y Phelan, 1995: 82). Por ello, una estrategia de atención a la salud que haya sido diseñada para incidir única y exclusivamente sobre factores de riesgo aislados, por más efectiva que sea en el corto plazo, no podrá garantizar a lo largo del tiempo resultados positivos, sino se acompaña de otras estrategias aún mayores, dirigidas a modificar mecanismos causales subyacentes a dichos factores, como pueden ser por ejemplo, las prácticas y relaciones de exclusión o explotación de las cuales se nutren las desigualdades sociales (Link, Phelan y Tehrainfar, 2010).

Si bien, la tesis de las causas fundamentales se formuló a mediados de la década de los 90, la evidencia empírica que la sustentó había estado circulando un par de décadas antes en trabajos que, en el contexto anglosajón, analizaban cómo la estratificación y las clases sociales condicionaban la reproducción de determinados patrones de mortalidad $y$ morbilidad $^{4}$. En el caso Británico, resalta el Reporte Black elaborado por el entonces Departamento de Salud y Seguridad Social de Gran Bretaña a inicios de 1980 (Blane, 1985; Smith, Bartley y Blane, 1990; MacIntyre, 1997), así como, los estudios longitudinales Whitehall I y II aplicados a distintas cohortes de empleados y empleadas del ayuntamiento de Londres (Marmot,

4 Cabe aclarar que en los trabajos de salud pública mencionados en este artículo, el uso del término clase social no se apega en estricto sentido a teorías sociológicas de orientación marxista o weberiana, sino que más bien se inspiran en aproximaciones relativas a jerarquías de estatus socioeconómicos. Para entender distinciones relativas a diferentes teorías de clase social véase: Grusky, 2008.
Rose, Shipley y Hamilton, 1978; Marmot, Shipley y Rose, 1984; Marmot, Smith, Stansfeld, Patel, North, Head, White, Brunner y Feeney, 1991; Marmot y Brunner, 2005; Stringhini, Berkman, Dugravot, Ferrie, Marmot, Kivimaki $y$ Singh-Manoux, 2011). Asimismo, en el contexto norteamericano se cuentan el informe sobre promoción de la salud elaborado por Marc Lalonde (1974), así como, el programa de Salud Poblacional (Population Health) fundado en 1987 por el Instituto Canadiense de Investigaciones Avanzadas (CIFAR-Canadian Institute for Advanced Research), el cual luego de su cierre en el 2001, daría lugar al programa Sociedades Exitosas (Evans, Barer y Marmor, 1994; Hall y Lamont, 2010).

A grosso modo, lo interesante de estos estudios y programas de investigación subyace en la distinción que introdujeron entre privaciones absolutas y relativas. No solo aquellos grupos sociales que experimentaban situaciones de privación absoluta (por ejemplo, una persona pobre, como podría esperarse) resultaron ser más propensos a desarrollar problemas de salud. Esta misma disparidad se repetía sistemáticamente conforme las posiciones socioeconómicas mejoraban, señalando la existencia de dinámicas de privación relativa entre aquellos grupos más aventajados del conjunto social, que tomadas en conjunto configuraban gradientes sociales: quienes tenían mayores niveles de ingreso y mejores tipos de ocupación presentaban progresivamente menores porcentajes de muerte (sin importar su causa específica) y menores probabilidades de desarrollar enfermedades crónicas como las de tipo cardiovascular (situación que ha seguido manteniéndose en el contexto británico en las últimas tres décadas).

El 'descubrimiento' de gradientes sociales supuso, por decirlo de alguna manera, el 'redescubrimiento' de la importancia que tienen las desigualdades en la configuración de estados de salud, algo que hasta ese momento no había sido debidamente sistematizado ni analizado comparativamente a lo largo de distintas sociedades. Asimismo, la renovada atención otorgada al tema de las desigualdades permitió justificar la necesidad de diseñar políticas sociales capaces de incorporar 
la dimensión institucional como un requisito importante para contextualizar los vínculos tradicionalmente establecidos entre estilos de vida, factores de riesgo aislados y enfermedades. Como se verá a continuación, la importancia que tienen las dinámicas institucionales en el campo de la salud pública ha sido una preocupación central presente en el debate global generado en torno a los llamados determinantes sociales de la salud.

\section{A PROPÓSITO DE LOS DETERMINANTES SOCIALES DE LA SALUD}

Actualmente, es en el debate sobre "determinantes sociales de la salud", en el cual el tema de los gradientes sociales ha logrado alcanzar mayor notoriedad y junto a ello, el argumento de que las desigualdades sociales deben ser entendidas como causas fundamentales de diversos estados de salud. La noción de determinante social fue adoptada inicialmente en la primera edición de The Solid Facts, trabajo editado en 1998 por Michael Marmot y Richard Wilkinson bajo el patrocinio de la Organización Mundial de la Salud (OMS). Dicho texto expuso diez lecciones básicas de salud pública aprendidas de países con diferentes niveles de desarrollo económico ${ }^{5}$ :

1) En una jerarquía de niveles socioeconómicos, conforme se va ascendiendo, la expectativa de vida crece y la probabilidad general de desarrollar enfermedades decrece (esta es la idea de gradiente social).

2) Prácticas y relaciones sociales que reproducen sistemáticamente situaciones de estrés, generan estados de ansiedad y preocupación que dañan los estados de salud individual y pueden conducir a muertes prematuras.

3) El acceso público o privado a redes de cuido, apoyo y educación a edades tempranas, incrementa la posibilidad de gozar de una buena salud en el transcurso de la vida.

Resumido de Wilkinson y Marmot, 2003.
4) El sufrir situaciones de exclusión social y discriminación socava en términos generales, la calidad de vida que puede gozar una persona, así como, los recursos a los cuales puede echar mano para lidiar con problemas de salud.

5) Mercados de trabajo que reproducen lógicas de inestabilidad e inseguridad laboral, aumentan la aparición de enfermedades en las poblaciones trabajadoras. Quienes acceden a puestos laborales estables $y$ pueden a su vez alcanzar un mayor grado de autonomía en sus actividades, tienden a presentar mejores estados de salud.

6) Altos niveles de desempleo, inciden en la aparición de enfermedades y muertes prematuras en el conjunto social.

7) La pertenencia a redes sociales solidarias en el ámbito laboral y de la comunidad, ayuda a mejorar el bienestar, tanto material como afectivo de las personas involucradas.

8) El abuso y dependencia de sustancias como el alcohol, drogas y tabaco aumenta en relación a las situaciones de exclusión social y discriminación que experimente la persona.

9) Tanto la provisión de alimentos a la cual tiene acceso una persona, como su calidad en términos nutricionales, varía en relación con la posición ocupada en una jerarquía socioeconómica. Por ejemplo, personas con menores ingresos tienden a remplazar el consumo de alimentos frescos con comidas procesadas $y$ de menor costo.

10) Aquellas ciudades que con el paso del tiempo, han logrado establecer formas de transporte público menos contaminante (como el uso de bicicletas), logran mejorar sus indicadores de bienestar general.

La popularidad de este primer informe, "probablemente uno de los textos de salud pública más influyentes e importantes en las últimas décadas" (Benach, 2009), se acentuó con la publicación de Social Determinants of Health, libro en el cual nuevamente Wilkinson y Marmot junto a su equipo de la University College 
London, presentaron resultados de estudios sobre clase social, pobreza y mercados laborales, entre otros, que vinieron a complementar con mayor evidencia empírica al decálogo de lecciones mencionadas anteriormente (Marmot y Wilkinson, 1998 y 2006) $)^{6}$. Más importante aún para la popularización del tema de los determinantes, fue la fundación en el año 2005 de la Comisión sobre Determinantes Sociales de la Salud (CDSS) por parte de la Organización Mundial de la Salud (OMS), comisión de la cual precisamente Michael Marmot fue director durante sus primeros tres años de formación (véase: CDSS, 2005).

Hasta la fecha, esta comisión ha sido la principal entidad encargada de difundir globalmente, el tema de las desigualdades sociales en el campo de la salud pública. Sus informes han tenido la virtud de convocar a un número mayor de actores, incluyendo no solo expertos de organizaciones de las Naciones Unidas, sino también a las llamadas redes de conocimiento (grupos de investigación científica), sectores políticos, así como, representantes de la sociedad civil $y$ diversos organismos no gubernamentales locales e internacionales. Más aún, el principal leitmotiv de este trabajo colectivo, el cual afirma que las inequidades sociales estaban cobrando vidas a gran escala $y$ que por tanto, su reducción debía convertirse en uno de los mayores imperativos éticos contemporáneos (CDSS, 2008), ha devenido desde entonces en un mantra para quienes apoyan $y$ dan vida al trabajo de la CDSS.

Al sostener que determinadas deudas en materia de justicia social, constituyen un asunto de vida o muerte, la CDSS permitió trasladar la discusión normativa sobre la salud y la enfermedad desde un ámbito de moral responsabilidad individual (el promovido desde encuadres biomédicos, tal y como fue comentado anteriormente), a uno de responsabilidad colectiva. En materia de diseño de políticas

$6 \quad$ Otros tópicos tratados fueron los de exclusión racial y étnica, la configuración de redes sociales y el capital social, el desarrollo de enfermedades en diferentes etapas a lo largo de la vida, así como, los puntos anteriormente señalados sobre transporte $y$ alimentación (Marmot y Wilkinson, 2006). sociales, lo anterior ha permitido sostener que las inequidades en salud no se resuelven únicamente al elevar o hacer más eficiente los gastos y cobertura médica, o al mejorar su nivel de sofisticación técnica (por más relevante que esto sea, obviamente). Se requiere que los sistemas públicos de salud sean parte de una colectividad capaz de poner en marcha acciones $y$ programas cuyos objetivos estén dirigidos a orientar el funcionamiento institucional en favor del bienestar colectivo.

\section{DISTINGUIENDO LAS CONDICIONES MATERIALES DE LAS PSICOSOCIALES}

En resumidas cuentas, la discusión sobre determinantes sociales de la salud ha establecido que la posición ocupada en la sociedad constituye una causa fundamental de la salud $y$ la enfermedad. Con ello, distingue aquellos determinantes que a nivel de un paciente individual, han marcado el desarrollo de una enfermedad (los cuales son analizados y tratados desde la medicina clínica), de aquellos determinantes que a nivel institucional, marcan el desigual desarrollo de patrones colectivos de morbilidad (y que hacen que, por citar un ejemplo, en una consulta médica se reciban más pacientes de una zona geográfica que de otra).

Prosiguiendo con esta línea argumentativa, cabe preguntarse por la 'naturaleza' de la relación de causalidad que se está postulando entre posición social y estado de salud: dentro de una determinada posición ¿qué hace que las personas allí situadas tengan o no más riesgo de enfermarse? Independiente de si dicha posición se ha configurado con base en prácticas y relaciones de desigualdad tan disímiles como las de clase, género o etnia, es posible identificar junto con Bartley (2004) y Carpiano, Link y Phelan (2008), al menos, dos tipos de interpretación sobre esta relación de causalidad, una referida a "condiciones materiales" y otra versada sobre "condiciones psicosociales".

El primer tipo de interpretación es la más común. Parte de la vieja presunción de que la falta de recursos tangibles para subsistir (alimentos adecuados, servicios básicos o dinero), incide directamente en el deterioro de los estados de la salud que reporta una población. Acá 
se establece un vínculo causal entre posición social $y$ estado de salud que es de corte materialista, en donde precisamente, el menoscabo del bienestar material se vincula con la aparición de ciertas enfermedades. Este punto de vista fue retomado en trabajos como el Reporte Black o los estudios Whitehall en Gran Bretaña, al momento de identificar y reconstruir gradientes sociales. Variables usadas para reconstruir jerarquías socioeconómicas, tales como, ingreso económico, nivel de escolaridad o tipo de ocupación, son tomadas como los principales predictores de patrones de morbilidad $y$ mortalidad reportados por una población. De esta manera, por ejemplo, la opción de tener un trabajo formal estable del cual devengar un buen salario, constituye un factor importante, tanto para cubrir necesidades básicas (alimentarse o poder habitar en un sitio apropiado), como para poder costear una adecuada atención médica cuando sea requerido?

La segunda interpretación es de tipo psicosocial. Sin negar la importancia del acceso a recursos tangibles, este tipo de interpretación enfatiza que las posiciones sociales generan efectos psicológicos, que a la postre son los que verdaderamente minan los estados de salud individual. Las covariaciones que puedan registrarse entre la carencia de recursos y la presencia de una enfermedad, median dichos efectos psicológicos. Se enfatiza en el análisis de las experiencias subjetivas adversas que van configurándose en dichas posiciones, así como, en la medición de las respuestas biológicas y emocionales que las mismas generan en las personas, como por ejemplo, niveles de ansiedad y estrés o bien, sentimientos de menosprecio, irrespeto, humillación o estigmatización ${ }^{8}$. La interpretación de orden psicosocial se encuentra representada en dos textos clave de la literatura sobre determinantes sociales de la salud: The status syndrome. How social standings affects our health and longevity de Michael Marmot (2004)

$7 \quad$ Ejemplos de modelos de análisis basados en interpretaciones materialistas se encuentran en Bartley, 2004, capítulo 6.

Ejemplos de modelos de análisis basados en interpretaciones psicosociales se encuentran en Bartley, 2004, capítulo 5. y Unhealthy societies: the afflictions of inequality de Richard Wilkinson (1996).

El primero resume mucho de las investigaciones que hasta ese momento Marmot había venido realizando sobre el tema de gradientes sociales. Desde su punto de vista, una vez resuelta la satisfacción de necesidades básicas, tales como: alimentación y la ubicación dentro de un contexto estratificado, determina variaciones de estatus, relativas a las recompensas (no necesariamente monetarias) que una persona obtiene del ocupar una posición social, tales como, prestigio, respeto, felicidad o conexiones interpersonales. Los estatus —esto sería lo más importante- les proporcionan a las personas diferentes oportunidades de alcanzar un tipo específico de bienestar que el autor denomina "autonomía", entendida como "el nivel de control que una persona tiene sobre su vida" $y$ sus opciones de participar en una existencia colectiva enriquecedora a nivel personal (Marmot, 2004: 2). Las experiencias negativas que pueden derivarse del estatus social y la falta de autonomía, son asimiladas por el cuerpo en términos de estados de estrés crónico que con el paso del tiempo, deterioran órganos clave para el funcionamiento biológico, en especial, el cerebro. En este sentido, la tesis central de The Status Syndrome puede resumirse de la siguiente manera: sociedades 'poco sanas' debido a sus desigualdades sociales, dan lugar a mentes estresadas que crean cuerpos enfermos ${ }^{9}$.

Por su parte, Richard Wilkinson logró fundamentar una interesante hipótesis en donde afirmaba que al comparar distintos países, aquellos en donde se había logrado sobrepasar un PIB per cápita equivalente a los us $\$ 5000$, la correlación entre niveles absolutos de ingreso y expectativa de vida perdía fuerza. Una vez alcanzados los Us $\$ 5000$ (momento en el cual las sociedades generalmente inician su transición epidemiológica ${ }^{10}$, las diferencias que

$9 \quad$ Para una síntesis sobre cómo se encuentran entrelazados las desigualdades sociales y los procesos fisiológicos relativos al estrés, véase Brunner y Marmot, 2006 y Krieger, 2012: 191-201.

10 La transición epidemiológica indica el momento en el cual en un conjunto social, las enfermedades crónicas empiezan a prevalecer sobre las infecto- 
mostraron los países en cuanto a expectativa de vida, empezaron a asociarse en mayor grado a las disparidades en sus respectivos niveles relativos de ingreso (Wilkinson, 1996: 34). Por ello, si bien es cierto, un país puede mejorar sus indicadores básicos en salud mediante alzas en el crecimiento económico, dicha mejoría no podrá continuar si ese crecimiento no se hace acompañar también por una reducción de las desigualdades económicas.

Para Wilkinson, las brechas de ingreso que se generan a lo interno de una sociedad, conducen no solo a la generación de estrés, sino también al deterioro de sus niveles de confianza y cohesión social, así como, al aumento de situaciones de conflicto social e inseguridad, todos estos factores que están en la base de la aparición de distintas patologías (1996: 176). En épocas más recientes, este autor ha continuado aportando información que sugiere que estas dinámicas de desintegración social afectan la salud de las personas directamente, al inducir sentimientos de marginalidad, hostilidad o depresión (Wilkinson, 2005; Wilkinson y Pickett, 2010).

\section{PERSPECTIVAS CRÍTICAS SOBRE LOS} DETERMINANTES SOCIALES DE LA SALUD

Sin lugar a dudas, el tema de los determinantes sociales enriqueció el espectro de discusión sostenido en diversas agendas de salud pública; sin embargo, la manera en que ha sido trabajado desde la CDSS, no se ha visto exenta de críticas. Sobre esto, hay al menos un punto por considerar brevemente en el marco de la revisión hecha en el presente artículo: la falta de 'politización' que predomina en gran parte del debate sobre determinantes.

Quizá sea Vicente Navarro, reconocido politólogo y economista en el campo de la salud, quien ha resumido de mejor manera el reclamo sobre 're-politización' generada en torno a los determinantes sociales de la salud. Como se mencionó anteriormente, el leitmotiv por excelencia del primer informe de la CDSS, rezaba que la inequidad social estaba cobrando vidas, matando gente a gran escala. En cambio, para Navarro

contagiosas "no son las desigualdades las que matan", sino aquellas personas y agrupaciones "responsables de dichas desigualdades" (2009: 423). Con ello, subraya algo que apenas había sido tocado en los informes de la CDSS, a saber, que los determinantes sociales son el resultado de actores e intereses públicos y privados concretos, con nombres $y$ apellidos, así como de las tensiones y relaciones de poder que los mismos han ido forjando en contextos históricos específicos.

El reclamo de Navarro hace eco del de otros pensadores críticos como David Coburn (Coburn, Poland, Robertson y Eakin, 1998; Coburn, 2000), Joan Benach y Carles Muntaner (2005 y Benach, 2009), quienes han puntualizado que la evolución actual de gradientes sociales y brechas en salud forman parte de una época signada por poco más de tres décadas de implementación de ideales y políticas de corte neoliberal. Desde este punto de vista, las explicaciones usualmente ofrecidas por la CDSS quedan cortas en tanto, no señalan cómo los disímiles patrones en morbilidad y mortalidad que han sido reportados, obedecen a cambios estructurales inducidos por clases económicas y políticas en distintas latitudes, como por ejemplo, la introducción del llamado 'nuevo gerenciamiento público' e iniciativas de privatización en los sistemas de salud, el desarrollo de discursos en los cuales los pacientes son asumidos en términos de clientes y consumidores, los recortes en los niveles de gasto o inversión social en salud, o la reducción de prestaciones universales de servicios médicos en favor del incremento de servicios basados en pago por aseguramiento. Dependiendo del caso que se tenga en mente, iniciativas como las citadas podrían ser consideradas entonces (y discúlpese acá el juego de palabras) como los determinantes de los determinantes sociales que enferman a las personas y sus sociedades.

En Latinoamérica, el llamado de atención sobre lo que podrían denominarse "determinantes político-económicos" de la salud, ha sido puesto de manifiesto por dos importantes corrientes de pensamiento en el campo de la salud pública: la "medicina social", desarrollada en el contexto hispano-parlante, y la llamada "salud colectiva" en Brasil (Waitzkin, 2001; 
López y Peña, 2006; Silva, 2011). Al hacer evidentes distintos problemas sufridos por diversos grupos sociales tradicionalmente excluidos en la región, mediante el uso de categorías analíticas de inspiración marxista (en su gran mayoría), ambas corrientes han podido no sólo plantear discursos alternativos al biomédico, sino también criticar el rumbo actual que han tomado los sistemas de salud de la región (Breilh y Granda, 1989; Breilh, 2003 y Granda, 2008). Actualmente, ambas corrientes de pensamiento se encuentran amparadas por la labor que desde 1984, ha venido realizando la Asociación Latinoamericana de Medicina Social (ALAMES), bajo el auspicio de la Organización Panamericana de la Salud (OPS).

Si bien, la vitalidad del debate crítico latinoamericano en torno al vínculo entre desigualdades sociales y estados de salud, aún no ha sido igualmente respaldada por una rigurosa $y$ voluminosa documentación y producción de datos empíricos comparativos ${ }^{11}$ (un poco a la inversa de lo sucedido en el contexto anglosajón, que se gana en producción de dato pero se pierde en capacidad crítica), sus principales llamados de atención con respecto al estudio de los determinantes sociales de la salud puede resumirse de la siguiente manera: a) reclamar que los análisis sobre este tema sean debidamente contextualizados en el marco de países cuyos procesos históricos mostrarán distintas especificidades político económicas; b) situar en estos contextos históricos las recomendaciones de política pública que se elaboran en los trabajos de la CDSS, para examinar y valorar sus posibilidades reales de implementación ${ }^{12}$

11 Véase por ejemplo el texto de Muntaner, Rocha, Borrell, Vallebuona, Ibañez, Benach y Sollar (2012) sobre la cantidad de artículos publicados en revistas electrónicas en relación con el tema de clase social y salud. Asimismo, López y Peña (2006: 90) concuerdan en afirmar que en Latinoamérica, la producción de investigaciones que integren lo social en el campo de la salud pública, "tiene un desarrollo joven".

Sobre este punto en particular, se ha señalado que una importante debilidad del primer informe de la CDSS consiste en haber obviado el por qué de las iniciativas promovidas anteriormente por la OMS, en relación con la necesidad de atender las y finalmente, c) acompañar la demanda por justicia social introducida en el debate sobre determinantes sociales de la salud, con una discusión acerca de la cuota de responsabilidad que tienen distintos grupos de poder económico y político en cuanto a la toma de decisiones que afectan la salud de distintas poblaciones en general (Birn, 2009; López y Peña, 2006).

\section{CONCLUSIÓN}

La manera en que las sociedades se organizan en torno a arreglos distributivos, así como, sus respectivas relaciones de exclusión y cierre social que les dan forma, condicionan la dispar capacidad o libertad que tienen sus miembros de sacarle provecho a distintos tipos de recursos necesarios para alcanzar y mantener estados de salud adecuados. El debate sobre determinantes sociales de la salud, sea en su versión de condicionantes materiales o psicosociales, ha contribuido a explicar que los patrones disímiles en morbilidad y mortalidad que presentan distintos grupos sociales se hallan condicionados por factores de estratificación, que no se derivan meramente de la mera voluntad o el desempeño individual. Como fue expuesto anteriormente, es necesario reconocer que el 'descubrimiento' de los gradientes sociales supuso a su vez el 'redescubrimiento' de la importancia que tienen las instituciones sociales en el campo de la salud pública.

Sin menoscabar en lo más mínimo, la importancia contemporánea que tiene el debate sobre determinantes sociales, se tienen los análisis de los vínculos existentes, por un lado, entre salud $y$ enfermedad $y$ por el otro, el de las desigualdades sociales e instituciones; análisis que necesitan ser profundizados y sometidos a distintas perspectivas críticas. El llamado de atención que se ha venido efectuando desde la salud colectiva como de la medicina social, constituye un primer buen ejemplo. Otras tareas aún pendientes de discutir serían el

desigualdades sociales para mejorar la salud de las poblaciones, que al cabo de los años no lograron llegar a buen puerto. Este sería, por ejemplo, el caso de la declaración de Alma-Ata de 1978, sobre el tema de atención primaria en salud (Birn, 2009). 
desarrollo de análisis de tipo relacional, capaz de situar gradientes sociales en distintas intersecciones de clase, género, ciudadanía y etnia; así como también, la utilización de métodos de investigación cualitativa para explorar las experiencias de vida de quienes ocupan esos espacios, para comprender de esta manera, desde su punto de vista, las razones por las cuales han tenido o no la capacidad de acceder y movilizar recursos para mejorar su estado de salud. Por lo pronto, estos dos últimos temas que trascienden los límites del presente artículo, quedarán pendientes de explorar en próximos trabajos.

\section{REFERENCIAS BIBLIOGRÁFICAS}

Bartley, Mel. Health inequality. An introduction to theories, concepts, and methods. Cambridge: Polity Press, 2004.

Breilh, Jaime. Epidemiología crítica. Ciencia emancipadora e intercultural. Buenos Aires: Lugar Editorial, 2003.

Breilh, Jaime y Granda, Edmundo. "Epidemiología y contra-hegemonía". Social Science and Medicine 28 (11). 1989: 1121-1127.

Benach, Joan y Muntaner, Carles. Aprender a mirar la salud. Cómo la desigualdad social daña nuestra salud. Barcelona: El viejo topo, 2005.

Benach, Joan. "Los determinantes sociales $y$ las desigualdades en salud: una visión crítica del Informe de la Comisión de Determinantes Sociales en Salud de la OMS". [Ponencia presentada en las Primeras Jornadas sobre Desigualdades Sociales en la Salud de la Comunidad de Madrid]. Madrid. 12 y 13 de noviembre 2009.

Birn, Anne-Emanuelle. "¿Politizando o puliéndolo? Subsanar las desigualdades en una generación: alcanzar la equidad sanitaria actuando sobre los determinantes sociales de la salud". Medicina Social 4 (3). 2009: 189-201.

Blane, David. "An assesment of the Black Report's explanations of health inequalities". Social Health and Illness 7 (3). 1985: 231-264.

Brunner, Eric y Marmot, Michael. "Social organization, stress, and health". Social determinants of health. Michael Marmot y Richard Wilkinson (eds.). Oxford: Oxford University Press, 2006.

Carpriano, Richard; Link, Bruce y Phelan, Jo C. "Social inequality and health: future directions for the fundamental cause explanation". Social class, how does it work? Annette Lareau y Dalton Conley (eds.). New York: Russel Sage Foundation, 2008.

Comisión sobre Determinantes Sociales de la Salud (CDSS). Action on the social determinants of health: learning from previous experiences. Ginebra: Comisión sobre Determinantes Sociales de la SaludOrganización Mundial de la Salud, 2005.

Comisión sobre Determinantes Sociales de la Salud (CDSS). Subsanar las desigualdades en una generación. Alcanzar la equidad sanitaria actuando sobre los determinantes sociales de la salud. Ginebra: Comisión sobre Determinantes Sociales de la Salud-Organización Mundial de la Salud, 2008.

Coburn, David. "Income inequality, social cohesion and the health status of populations: the role of neo-liberalism". Social Science and Medicine 51. 2000: 135-146.

Coburn, David; Poland, Blake; Robertson, Ann y Eakin, Joan. "Wealth, equity and health care: a critique of a 'population health' perspective on the determinants of health". Social Science and Medicine 46. 1998: 785-798.

Cockerham, William. Medical sociology. $12^{\text {th }}$ edition. Boston, MA: Prentice Hall, 2012.

Cockerham, William. Social causes of health and disease. Malden, MA: Polity, 2008.

Evans, Robert; Barer, Morris y Marmor, Theodore. Why are some people healthy and others not? The determinants of health of populations. New York: Aldine de Gruyter, 1994.

Granda, Edmundo. "Algunas reflexiones a los veinticuatro años de la ALAMES". Medicina Social 2 (3). 2008: 217-225.

Grusky, David. Social stratification: class, race, and gender in sociological 
perspective. $3^{\text {rd }}$ edition. Boulder, co: Westview Press, 2008.

Hall, Peter y Lamont, Michèle (eds.). Succesful societies. How institutions and culture affect health. Cambridge: Cambridge University Press, 2010.

Krieger, Nancy. Epidemiology and the people's health. Theory and context. Oxford: Oxford University Press, 2012.

Lalonde, Marc. A new perspective on the health of Canadians. Ottawa: Ministry of Health and Welfare, 1974.

Link, Bruce y Phelan, Jo C. "Social conditions as fundamental causes of disease". Journal of Health and Social Behaviour 35 (S). 1995: 80-94.

Link, Bruce y Phelan, Jo C. "Understanding sociodemographic differences in healthThe role of fundamental causes". Editorial. American Journal of Public Health 86 (4). 1996: 471-473.

Link, Bruce; Phelan, Jo C. y Tehranifar, Parisa. "Social conditions as fundamental causes of health inequalities: theory, evidence, and social implications". Journal of Health and Social Behaviour 51 (S). 2010: 28-40.

López Arellano, Olivia y Peña, Florencia. "Salud y sociedad. Aportaciones del pensamiento latinoamericano". Medicina Social 1 (3). 2006: 82-102.

MacIntyre, Sally. "The Black Report and beyond. What are the issues?". Social Science and Medicine 44 (6). 1997: 723-745.

Marmot, Michael. "Social causes of social inequalities in health". Public Health, Ethics, and Equity. S. Anand, F. Peter y A. Sen. (eds.). Oxford: Oxford University Press, 2004.

Marmot, Michael. The status syndrome. How social standing affects our health and longevity. New York: Holt Paperbacks, 2005.

Marmot, Michael y Brunner, Eric. "Cohort profile: the Whitehall II study". International Journal of Epidemiology 34. 2005: 251-256.

Marmot, M.; Rose, G.; Shipley, M. y Hamilton, P. "Employment grade and coronary heart disease in British civil servants". Journal of Epidemiology and Community Health 32 (4). 1978: 244-249.

Marmot, Michael; Shipley, Martin y Rose, Geoffrey. "Inequalities in death-specific explanations of a general pattern". Lancet 323 (8384).1984: 1003-1006.

Marmot, M.; Smith, G.; Stansfeld, S.; Patel, C.; North, F.; Head, J.; White, I.; Brunner, E. $y$ Feeney, A. "Health inequalities among British civil servants: the Whitehall II study". Lancet 337 (8754). 1991: 1387-1393.

Marmot, Michael y Wilkinson, Richard (eds.). Social determinants of health. $1^{\text {st }}$ edition. Copenhaguen: World Health Organization, 1998.

Marmot, Michael y Wilkinson, Richard (eds.). Social determinants of health. $2^{\text {nd }}$ edition. Oxford: Oxford University Press, 2006.

Martínez, Ángel. Antropología médica. Teorías sobre la cultura, el poder y la enfermedad. Madrid: Anthropos, 2008.

Muntaner, Carles; Rocha, Katia; Borrell, Carme; Vallebuona, Clelia; Ibañez, Ciro; Benach, Joan y Sollar, Orielle. "Clase social y salud en América Latina". Revista Panamericana de Salud Pública 31 (2). 2012: 166-174.

Navarro, Vicente. "What we mean by social determinants of health". International Journal of Health Services 39 (3). 2009: 423-441.

Porta, Miquel. A Dictionary of Epidemiology. $5^{\text {th }}$ edition. Oxford University Press, 2008.

Saracci, Rodolfo. Epidemiology. A very short introduction. Oxford: Oxford University Press, 2010.

Silva, Jairnilson. Desafíos para la salud colectiva en el siglo XXI. Buenos Aires: Lugar Editorial, 2011.

Smith, George; Bartley, Mel y Blane, David. "The Black Report on socioeconomic inequalities in health 10 years on". British Medical Journal 301. 1990: 373-377. 
Stringhini, S.; Berkman, L.; Dugravot, A.; Ferrie, J. E.; Marmot, M.; Kivimaki, M. y Singh-Manoux, A. "Socioeconomic status, structural and functional measures of social support, and mortality. The British Whitehall II cohort study, 1985-2009". American Journal of Epidemiology 175 (12). 2011: 1275-1283.

Waitzkin, Howard. "Social medicine then and now: lessons from Latin America". American Journal of Public Health 91 (10). 2001: 1592-1601.

Wilkinson, Richard. Unhealthy societies: the afflictions of inequality. London: Routledge, 1996.
Wilkinson, Richard. The impact of inequality. How to make sick societies healthier. New York: The New Press, 2005.

Wilkinson, Richard y Marmot, Michael. The Solid Facts. Second Edition. Ginebra: Organización Mundial de la Salud-oms, 2003.

Wilkinson, Richard y Pickett, Kate. The spirit level: why greater equality makes society stronger. London: Penguin Books, 2010.

Fecha de ingreso: 23/08/2012 Fecha de aprobación: 28/01/2013 